\title{
BMJ Global Health Readiness and early response to COVID-19: achievements, challenges and lessons learnt in Ethiopia
}

Betty Lanyero, ${ }^{1}$ Zewdu Assefa Edea, ${ }^{2}$ Emmanuel Onuche Musa, ${ }^{3}$ Shambel Habebe Watare (10 , ${ }^{4}$ Mayur Lalji Mandalia, ${ }^{5}$ Martin Chibueze Livinus, ${ }^{1}$ Faiqa Kassim Ebrahim, ${ }^{1}$ Abiy Girmay, ${ }^{1}$ Aggrey Kaijuka Bategereza, ${ }^{1}$ Aschalew Abayneh, ${ }^{4}$ Boureima Hama Sambo, ${ }^{6}$ Ebba Abate ${ }^{7}$

To cite: Lanyero B, Edea ZA, Musa E0, et al. Readiness and early response to COVID-19: achievements, challenges and lessons learnt in Ethiopia. BMJ Global Health 2021;6:e005581. doi:10.1136/ bmjgh-2021-005581

Handling editor Seye Abimbola

Received 3 March 2021 Accepted 27 May 2021
Check for updates

(c) Author(s) (or their employer(s)) 2021. Re-use permitted under CC BY-NC. No commercial re-use. See rights and permissions. Published by BMJ.

For numbered affiliations see end of article.

Correspondence to

Dr Betty Lanyero;

lanyerob@who.int

\section{ABSTRACT}

Declaration of the novel coronavirus disease as a Public Health Emergency of International Concern necessitated countries to get ready to respond. Here, we describe key achievements, challenges and lessons learnt during the readiness and early response to COVID-19 in Ethiopia. Readiness activities commenced as early as January 2020 with the activation of a national Public Health Emergency Operations Centre and COVID-19 Incident Management System (IMS) by the Ethiopian Public Health Institute. The COVID-19 IMS conducted rapid risk assessments, developed scenario-based contingency plans, national COVID-19 guidelines and facilitated the enhancement of early warning and monitoring mechanisms. Early activation of a coordination mechanism and strengthening of detection and response capacities contributed to getting the country ready on time and mounting an effective early response. High-level political leadership and commitment led to focused efforts in coordination of response interventions. Health screening, mandatory 14-day quarantine and testing established for all international travellers arriving into the country slowed down the influx of travellers. The International Health Regulations (IHR) capacities in the country served as a good foundation for timely readiness and response. Leveraging on existing IHR capacities in the country built prior to COVID-19 helped slow down the importation and mitigated uncontrolled spread of the disease in the country. Challenges experienced included late operationalisation of a multisectoral coordination platform, shortage of personal protective equipment resulting from global disruption of importation and the huge influx of over 10000 returnees from different COVID-19-affected countries over a short period of time with resultant constrain on response resources.

\section{INTRODUCTION}

On 30 January 2020, the Director General of the WHO declared COVID-19 a Public Health Emergency of International Concern (PHEIC), and on 12 March 2020, it was declared a pandemic. ${ }^{1}$ This announcement inherently called for a coordinated

\section{Summary box}

Operational readiness to respond to an emergency is a critical element of the emergency management cycle.

- The setup of an incident management system (IMS to support readiness actions for an emergency. Ordinarily, an IMS is set up to support response activities; however, in this situation, it was set up to support readiness actions given the imminent threat of importation of COVID-19.

- High level of political commitment to coordinate response actions enabled a progressive multidisciplinary engagement of non-health sectors and private partnerships such as education, trade and industries, transport, culture and tourism and law enforcement bodies.

- Multisectoral collaborations and private partnerships have been vital to getting the country ready. Efforts to strengthen and maintain the collaboration and partnership are encouraged Strengthening country core capacities for health emergencies including pandemics in line with the International Health Regulations is essential.

international response due to the high risk of a spread and a step up of readiness capacities to respond to the pandemic.

With the outbreak of a new disease, determining Ethiopia's level of operational readiness to respond timely and effectively was essential. Operational readiness is critical for the identification of high priorities and imminent risks for which mitigation and response actions should be undertaken. ${ }^{23}$ State parties are required to develop and maintain public health emergency response capacities to detect, assess, notify and report any events or emergencies under the International Health Regulations (IHR) (2005) such as national legislation, policy and financing for public health events, coordination and national 
focal point communications, surveillance, risk communication, laboratory diagnostic and confirmation capacity and points of entry (PoE) among others. ${ }^{4}$ However, having these capacities does not necessarily mean one is ready to respond. WHO defines operational readiness as the outcome of preparedness actions such as planning, allocation of resources, training, exercising and organising to build, sustain and improve operational capabilities based on the risk assessment. ${ }^{5}$ A self-assessment using an operational readiness checklist for COVID- $19^{6}$ was conducted to determine level of operational readiness across the 47 countries under WHO Regional Office for Africa in February 2020. ${ }^{78}$ As detailed elsewhere, ${ }^{8}$ Ethiopia demonstrated adequate readiness capacities in coordination structures, case management, infection prevention and control (IPC), logistics, risk communication and community engagement and surveillance. Limited capacities were noted in laboratory capacity and rapid response teams. ${ }^{8}$ Similar readiness assessments conducted at subnational level showed wide-ranging levels of readiness capacities across the regional states.

This article documents the operational readiness and early response interventions embarked upon by the Government of Ethiopia (GoE) in the first 6 months from January 2020 for the COVID-19 pandemic. We discuss the challenges and lessons learnt which could be used to inform future management of health emergencies.

\section{OPERATIONAL READINESS FOR COVID-19}

Readiness measures undertaken in Ethiopia prior to detection of the first case of COVID-19 are categorised under three major thematic areas: (1) coordination and leadership, (2) enhancement of detection and response capacities and (3) strengthening of early warning mechanisms.

\section{Coordination and leadership}

A three-layered coordination structure was established to support the coordination of the COVID-19 readiness and response in Ethiopia: the strategic level coordination consisting of ministerial taskforces and chaired by His Excellency the Prime Minister; the multisectoral level coordination led by the National Disaster and Risk Management Committee (NDRMC) and the tactical level led by Public Health Emergency Operations Centre (PHEOC). At the operational level, PHEOCs were activated in all 12 regional states in the country. Early response action and mitigation measures were guided by the declaration of a state of emergency by the Prime Minister on 8 April 2020 after the first few cases were reported in the country. Schools, bars and night clubs were closed and mass gatherings were banned. ${ }^{9} 10$ Additionally, number of persons in vehicles was reduced by $50 \%$, and changes in work modalities with emphasis on the use of virtual platforms were made.

Activation of the national PHEOC and the COVID-19 readiness and response Incident Management System
(IMS) led by Ethiopian Public Health Institute (EPHI) was instrumental. EPHI oversees the preparedness and response mechanisms for any public health emergency in Ethiopia. As early as 27 January 2020, the national PHEOC was activated and the IMS established to coordinate and lead the public health readiness and response to COVID-19. The IMS led by the Minister of Health had pillars in surveillance, $\mathrm{PoE}$ and quarantine, case management, IPC, risk communication and community engagement, supplies and logistics and planning among others (figure 1). At the EPHI PHEOC, the incident management team in collaboration with WHO coordinated risk assessments, enhanced early warning mechanisms and guided the development of COVID-19-specific preparedness and response plans.

\section{Risk assessment}

Given the looming threat of COVID-19 to the country, rapid risk assessments for COVID-19 infection and spread in Ethiopia were conducted by WHO as early as January 2020. The risk was observed to be very high. As a result, Ethiopia was graded as one of the 13 'priority 1' countries in Africa with the need to prioritise readiness actions. At country level, risk assessments conducted by EPHI and WHO identified the risks of COVID-19 to human health, its spread and the control capacities with the available resources. One of such rapid risk assessments conducted on 6 February 2020 noted that there was a significant risk of importation and spread of COVID-19 to Ethiopia. At this time, there were no confirmed cases reported in Ethiopia and in the entire African continent. Ethiopia is one of the major transport hubs for Africa, with five daily direct flights to and from China which was the epicentre of COVID-19 at that point. Consequently, early mitigation measures were implemented at the PoE, capacities to detect suspect cases and quickly respond and risk communication messaging.

\section{Contingency planning}

Using a scenario-based approach, EPHI in collaboration with Federal Ministry of Health (FMOH), WHO, UN agencies and implementing partners developed the national COVID-19 Emergency Preparedness and Response Plan (EPRP) with an estimated budget of US $\$ 13$ million. ${ }^{11}$ The COVID-19-specific EPRPs were developed at regional levels and fed into the national plan. These plans were adjusted as the scenarios changed with the evolution of the outbreak. Incident action plans were developed at the level of the national and regional PHEOCs as early as January 2020. WHO conducted table-top simulation exercises (SIMEX) for the FMOH, EPHI, the United Nations in Ethiopia and other sector representatives to examine the readiness mechanisms in place.

The early preparations for strategies to maintain essential health services were recognised to prevent the potential impact of the diversion of health resources solely to a COVID-19 response. Hence, a taskforce on continuity of essential health services was set up under the leadership 


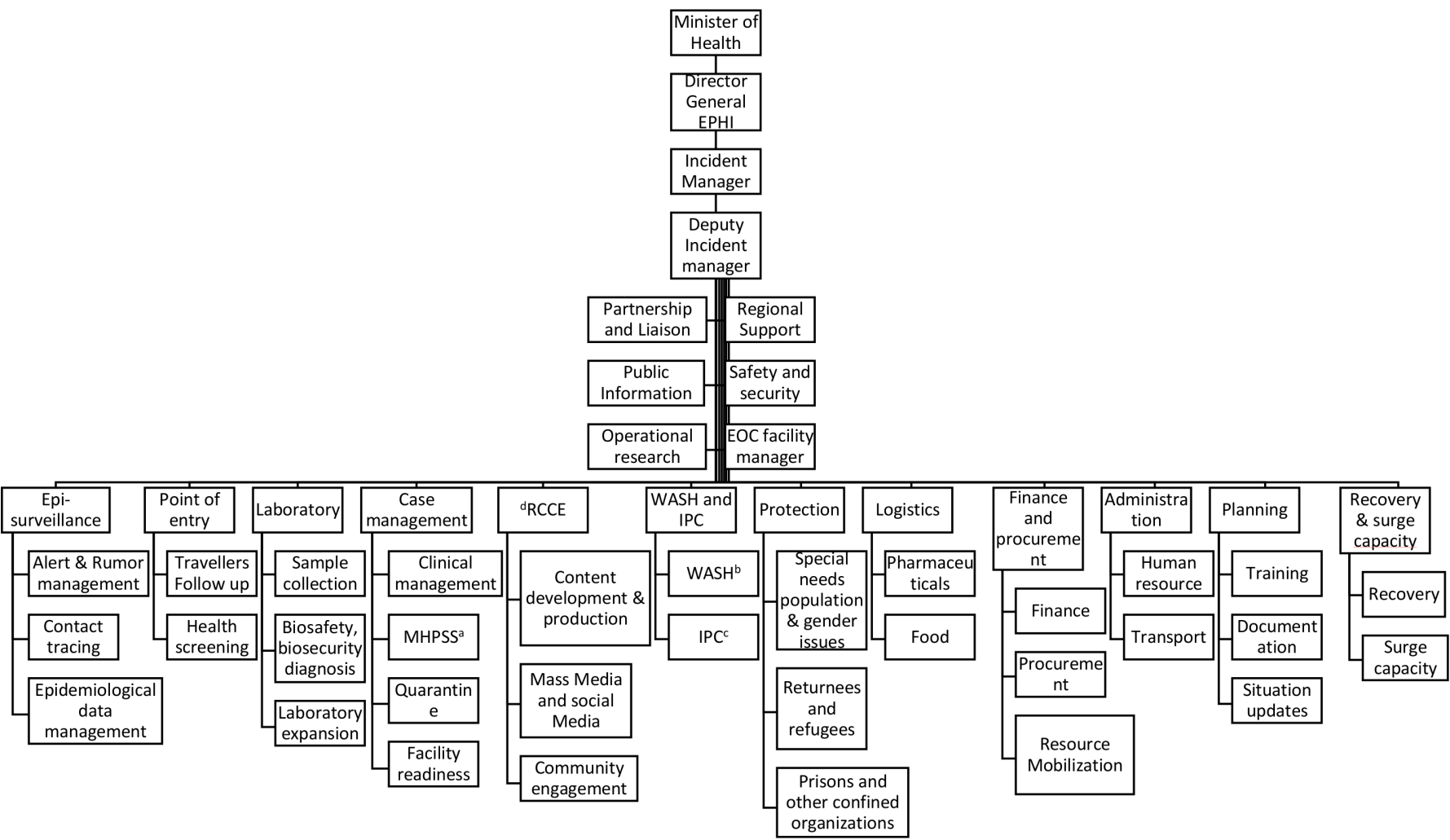

Figure 1 Structure of the COVID-19 Incident Management System (IMS) at the Ethiopian Emergency Operations Centre as of 30 March 2020. The COVID-19 IMS set up by Federal Ministry of Health and led by the Minister of Health Source consisted of 18 pillars with subunits. Based on their expertise, individuals were appointed to lead and coordinate readiness and response activities under each pillar. EPHI, Ethiopian Public Health Institute; IPC, infection prevention and control; MHPSS, mental health and psychosocial support; RCCE, risk communication and community engagement; WaSH, water, hygiene and sanitation,EOC, Emergency Operations Centre. Data source: EPHI.

of the State Minister of Health. The taskforce steered the preparation of health facilities and healthcare workers on the alternate referral pathways and access to treatment. Although the repurposing of human resources affected the delivery of health services with an increased workload, the continuity of essential health services including other disease outbreaks and health emergencies was prioritised.

\section{Enhancing detection and response capacity \\ Points of entry}

Interventions implemented at PoE aimed at the identification of ill travellers with symptoms and signs of COVID19 , identification of appropriate public health measures such as quarantine, isolation or treatment facilities at PoE, and provision of information to travellers on the public health risks and required precautionary measures during travel. Standard operating procedures and COVID-19 health declaration forms developed for PoE sites facilitated and enhanced health screening, information gathering and contact tracing for arriving passengers. Over 2700 Ethiopian Airlines supervisors, health screening staff, flight crew and support personnel were trained on COVID-19 early detection, isolation, prevention and response measures and communication on board aircrafts and in the airport in handling all travellers and suspected cases. A temporary isolation unit was established at Bole International Airport to isolate suspect cases at the airport before transfer to designated isolation facilities. With the increased spread of COVID-19 globally, several countries closed their borders and airline services; however, Ethiopian Airlines, the world's fourth largest airline by the number of countries served, maintained and increased cargo operations. This facilitated the transport of critical medical supplies to Ethiopia and to the rest of the African continent. Passenger operations to select countries continued which offered the possibility of bringing in more expertise to support the response.

\section{Laboratory capacities for COVID-19 testing}

Like almost all countries in Africa, Ethiopia did not have the capacity to test for COVID-19 at the onset. Samples were initially transported for testing to the National Institute for Communicable Diseases (NICD) Laboratory in South Africa, a WHO regional reference laboratory. With support from WHO and the Africa Centres for Disease Control and Prevention, EPHI set up the National Influenza and Arboviruses Reference Laboratory as the first COVID-19 testing laboratory in the country by 7 February 2020. Capacity building for laboratory technicians was conducted and donations of COVID-19 detection and extraction kits and reagents were made. WHO Ethiopia 
continued to support external quality control testing with samples shipped to NICD. Expansion of COVID-19 laboratory testing sites to the regional states was ramped up early in the response through the pre-existing laboratory network with regional public health laboratories, universities and laboratories under the Ministry of Agriculture.

Case management and infection prevention and control capacities Case management and IPC actions focused on identification and equipping the treatment and isolation facilities designated to receive COVID-19 cases, while building the capacity of required personnel to effectively manage COVID-19 cases. Among the initial steps undertaken were the adaptation of treatment and IPC protocols to the country context and setup of severe acute respiratory infections (SARI) treatment facilities in line with WHO recommendations. A standardised checklist was developed and used nationwide to assess facility readiness to identify gaps for improvement.

\section{Capacity building in COVID-19}

EPHI, in collaboration with WHO and partners, conducted training for over 10000 personnel at national and regional state levels. A training of trainers' course for 600 rapid response team members was conducted. Among those trained, were various cadres such as clinicians from major hospitals, personnel handling COVID-19 hotlines, PoE health practitioners, the disaster management assistant team, emergency medical team, laboratory professionals from regional laboratories, the federal police, defence force, special forces, central command and airforce, Ethiopian Airlines cabin crew members, hotel managers and the media among others.

\section{Operations, logistics and supplies}

Stockpile assessments conducted revealed urgent need to procure key medical supplies, personal protective equipments (PPEs) and laboratory items to support the country to at least respond to its first 100 cases. WHO Ethiopia donated over 1000 sets of PPEs from regional Ebola stockpiles in Uganda and Ghana. Simultaneously, in an exhaustive quantification exercise, the PHEOC logistics team identified the need for medical supplies based on planning assumptions. Often, a quick option to obtain supplies is through procurement from the local market; however, a local market survey conducted revealed that majority of the supplies could not be obtained in country. Thus, the procurement process for medical supplies was done internationally through the established global supply chain coordination cell platform coordinated by WHO in collaboration with UNICEF and partners. WHO mobilised supplies from stockpiles at regional hubs, the African Union and the GoE received donations from the Jack Ma foundation and other bilateral donors. The Ethiopia private sector also donated supplies to the country.

\section{Strengthening of early warning systems}

Early warning systems are intended to provide critical information for action. ${ }^{12}$ Early case detection, case investigation and timely referrals for treatment through enhanced surveillance mechanisms played a role in the containment of the disease early in the response. Alert and rumour management, contact tracing, active case search, community-based surveillance, sentinel site surveillance, mortality surveillance and PoE surveillance were some of the surveillance strategies that were enhanced. To bolster surveillance actions, technical guidance on the implementation of the surveillance strategies through capacity building, regular strategic guidance meetings and field visits were provided by EPHI and WHO. A strategy of conducting COVID-19 surveillance at SARI/influenzalike illness (ILI) sentinel surveillance sites also contributed to enhancing surveillance actions in Ethiopia. Since 2008, Ethiopia has established influenza hospital-based sentinel surveillance in eight sites in collaboration with WHO and United States Centers for Disease Control and Prevention. ${ }^{13} 14$ COVID-19 testing was conducted on ILI and SARI samples that were sent to the National Influenza and Arbovirus Reference Laboratory.

Enhancing risk knowledge management on COVID-19 was done through development and dissemination of daily situation reports containing epidemiological data analysis and readiness actions were produced and shared widely with partners, donors and the public by EPHI and Regional Health Bureau (RHB) in collaboration with WHO. ${ }^{15-17}$ The information products shared practices that could be used by other countries during this pandemic.

Risk communication messages were tailored for key populations to guide the actions undertaken by individuals, institutions and the community. National and international experts from EPHI, FMOH, UN agencies and partners were engaged to support develop a COVID-19 risk communication and community engagement strategy and operational plan. The strategy highlighted the protocols for communication flow, content development, press briefings, media scanning, community engagement, rumour management and capacity building. Information, education and communication materials were developed around public health measures, signs and symptoms of COVID-19 and actions to take if displaying specific symptoms. This information was translated into 12 local languages of Ethiopia and distributed to communities and health workers. Targeting of messaging was supported by the outcomes of an audience analysis to identify communication gaps as similarly emphasised by Lohiniva $e t \mathrm{al}^{18}$ in a study conducted in Finland on practical approaches to risk communication during epidemics. For example, through audience analysis in communities in Somali region, it was identified that some perceived that they were at minimal or no risk of acquiring COVID-19 because they are protected by their faith in Allah. Some communities perceived that those that contracted COVID-19 were those disobedient to the teaching of the holy Quran. To this end, key messages were developed focusing on creating awareness on the transmission of COVID-19 and the public and social measures to be implemented. Moreover, the Imams and other religious leaders were leveraged to disseminate these key messages. 
This led to notable behavioural changes among the community members such as wearing of face masks and hand hygiene. In addition, at the mosques, masks were worn and physical distancing measures were implemented.

\section{CHALLENGES}

The readiness and early response were faced by some key challenges. First, multisectoral coordination was weak. The existing multisectoral platform known as the emergency coordination committee chaired by the NDRMC and bringing together various government sectors and partners in emergency preparedness and response was not fully functional over a long period. This may be attributed to a limited understanding of the nature of COVID19, rumours of COVID-19 being a myth and the initial perception that it was predominantly a health issue. This broader engagement would have contributed to a whole-of-society approach in preparing for the pandemic and mounting a much stronger response. Second was the shortage of PPEs in the country resulting from the global supply chain disruption of the importation. Lack of PPE stockpiles in country and limited capacity for local production increased the risk of healthcare worker infections. Third, the huge influx of over 10000 returnees from different COVID-19-affected countries over a short period of time constrained response resources. For instance, additional quarantine and isolation facilities had to be established with provision of essential health services, mental health and psychosocial support and rehabilitation services. Fourth, the multiple health emergencies in the country, such as yellow fever, cholera, dengue, measles, disease outbreaks, flooding and population displacement, further strained the health system. The existing shortage of health workers, medical supplies and logistics was compounded by having to balance the needs between COVID-19 and non-COVID-19 response.

\section{LESSONS LEARNT}

The early setup of the PHEOC and the IMS enabled effective coordination of readiness actions and early response interventions. This was as early as 2 months before the first COVID-19 case was reported in the country. Ordinarily, an IMS is set up to support response activities; ${ }^{19}$ however, in this case, it was set up to support early readiness actions and effective timely response given the imminent threat of importation of the disease. Ethiopia also invested the 2-month interval between the time when COVID-19 was declared a PHEIC and when the first case was reported in the country to build the capacity of healthcare professionals in the management of COVID-19. The existence of trained human resources under the public health emergency management at the national, regional, district and health facility levels with basic surveillance skills facilitated the learning process.

We learnt that the high-level political leadership and commitment facilitated the efforts in ensuring a coordinated multisectoral readiness and early response interventions from strategic, technical and operational perspectives. This enabled a progressive multidisciplinary engagement of non-health sectors such as education, trade and industries, transport, culture and tourism and law enforcement bodies. The high political engagement is in line with global lessons learnt in the response to COVID-19 pandemic such as whole of government approach. ${ }^{20}$ Second, we also learnt that the IHR capacities built in the country prior to COVID-19 served as a good foundation for readiness and response to the pandemic. WHO plays a key role in supporting member states in building IHR capacities including the development

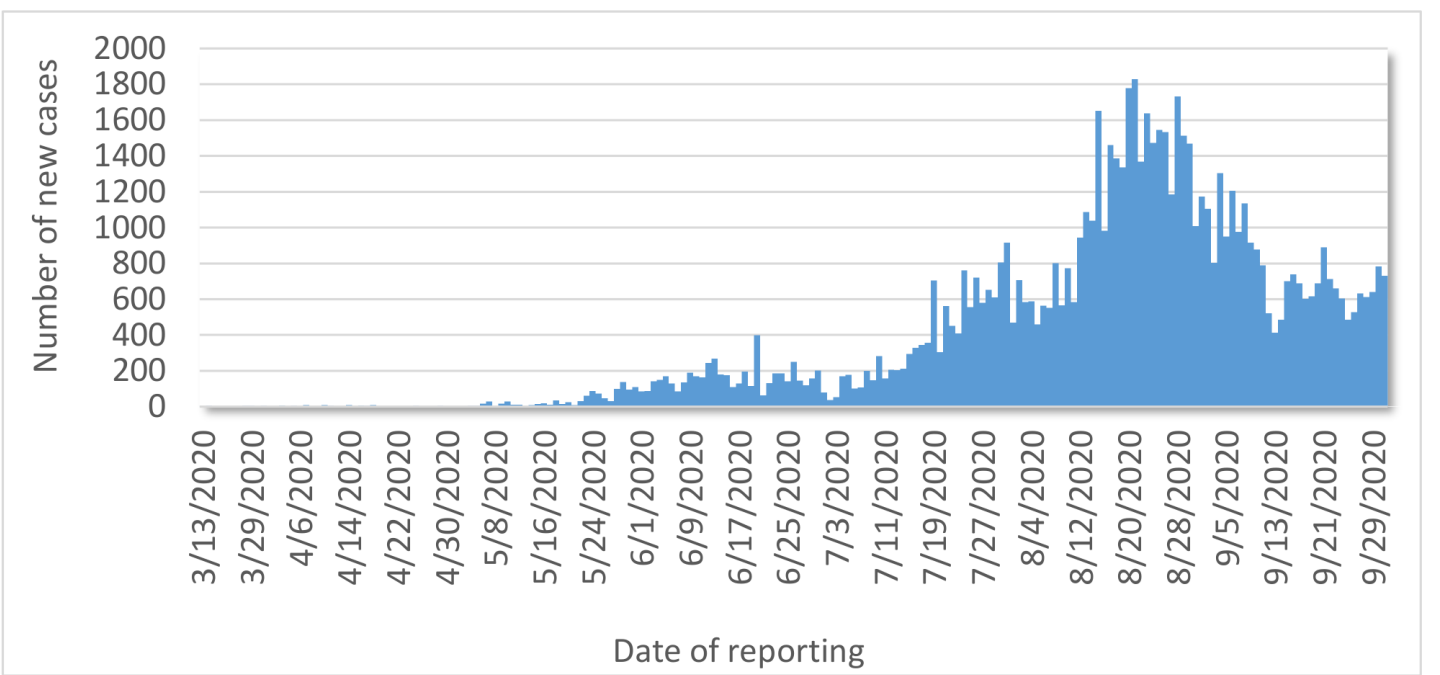

Figure 2 Epidemiological trend of confirmed COVID-19 cases in Ethiopia as of 29 September 2020. A bar chart showing the number of new confirmed COVID-19 cases per day starting from 13 March 2020 when the first case of COVID-19 was confirmed in Ethiopia. Data on the number of new cases were collected daily through the laboratory and surveillance pillars, compiled and used to develop the epidemiological trend. The epidemiological curve shows a slow and gradual increase in the COVID-19 confirmed cases in the first 4 months since March 2020 . The readiness and early response measures may have contributed to this trend. 
of National Action Plans for Health Security. In Ethiopia, critical IHR capacities in the areas of incident management, laboratory, PoE, surveillance and cross-border collaboration were already in place and were readily activated for this response. Third, the mandatory 14-day quarantine rule established on 23 March 2020 for all international travellers arriving into the country slowed down the influx of passengers and importation of the virus. It enabled the national authorities to prepare for and position resources to detect cases and rapidly isolate them. This may have led to a delayed peak for nearly 3 months following the confirmation of the first case. Fourth, private partnerships for emergency response contributed to expanding the capacity and leverage expertise in areas of case management and laboratory testing. Ethiopia collaborated with university institutions, private hospitals and laboratories for this purpose.

In conclusion, early operational readiness actions undertaken in Ethiopia contributed to the slowing down of the importation and spread of COVID-19 in the country. As observed, the epidemiological curve of the COVID-19 infection showed a gradual increase for nearly four months before cases started to peak (figure 2). The strong efforts to initiate readiness actions while on leveraging existing IHR capacities, coupled with high-level political commitment and leadership, helped mitigate the uncontrolled spread of the disease. Sustained efforts are recommended to control further transmission and minimise the health and socioeconomic impact on the population.

\section{Author affiliations}

${ }^{1}$ Emergency Preparedness and Response Unit, World Health Organization Country Office for Ethiopia, Addis Ababa, Ethiopia

${ }^{2}$ Early Warning and Information System Management Directorate, Ethiopian Public Health Institute, Addis Ababa, Ethiopia

${ }^{3}$ WHE Programme, World Health Organization Regional Office for Africa, Brazzaville, Republic of Congo

${ }^{4}$ Public Health Emergency Management, Ethiopian Public Health Institute, Addis

Ababa, Ethiopia

${ }^{5}$ Liaison Office to the African Union and the UN Economic Commission for Africa, World Health Organization, Addis Ababa, Ethiopia

${ }^{6}$ World Health Organization Representative to Ethiopia, World Health Organization Country Office for Ethiopia, Addis Ababa, Ethiopia

${ }^{7}$ Director General office, Ethiopian Public Health Institute, Addis Ababa, Ethiopia

Twitter Martin Chibueze Livinus @livimart

Contributors BL participated in the design and implementation of readiness and early response actions and drafted the manuscript. ZAE, EOM, SHW, MCL, FKE, $M L M, A G, A K B, A A, B H S$ and EA participated in the design and implemented the readiness and early response measures for COVID-19 pandemic in Ethiopia and reviewed the manuscript.

Funding The authors have not declared a specific grant for this research from any funding agency in the public, commercial or not-for-profit sectors.

Competing interests None declared.

Patient consent for publication Not required.

Provenance and peer review Not commissioned; externally peer reviewed.

Data availability statement All data relevant to the study are included in this work.
Open access This is an open access article distributed in accordance with the Creative Commons Attribution Non Commercial (CC BY-NC 4.0) license, which permits others to distribute, remix, adapt, build upon this work non-commercially, and license their derivative works on different terms, provided the original work is properly cited, appropriate credit is given, any changes made indicated, and the use is non-commercial. See: http://creativecommons.org/licenses/by-nc/4.0/.

ORCID iD

Shambel Habebe Watare http://orcid.org/0000-0002-5554-4027

\section{REFERENCES}

1 Archived: WHO Timeline - COVID-19. Available: https://www. who.int/news-room/detail/27-04-2020-who-timeline---covid-19 [Accessed 9 Jul 2020].

2 Tran BX, Hoang MT, Pham HQ, et al. The operational readiness capacities of the grassroots health system in responses to epidemics: implications for COVID-19 control in Vietnam. J Glob Health 2020;10.

3 Chan M. Learning from Ebola: readiness for outbreaks and emergencies. Bull World Health Organ 2015;93:818-818A.

4 WHO. International health regulations, 2005. Available: http://www. who.int/ihr/publications/9789241580496/en/ [Accessed 8 Jul 2020].

5 Layout C 3D by DMW. Strategic framework for emergency preparedness, 2017: 29.

6 WHO. Operational readiness checklist for COVID-19. Available: https://www.euro.who.int/_data/assets/pdf file/0004/428863/ Operational-Readiness-Checklist_final-version_Feb-13.pdf [Accessed 24 May 2021].

7 WHO. Novel Coronavirus (COVID-19) External Update \#1. Available: https://www.afro.who.int/sites/default/files/COVID-19\%20situation\% 20reports/COVID-19-Bulletin_18\%20Feb.pdf [Accessed 25 Sep 2020].

8 WHO. Daily Bulletin on COVID-19 - Readiness Situation Update WHO African Region. Available: http://whotogo-whoafroccmaster. newsweaver.com/JournalEnglishNewsletter/1d5f08bl78a [Accessed 25 Sep 2020].

9 Shigute Z, Mebratie AD, Alemu G, et al. Containing the spread of COVID-19 in Ethiopia. J Glob Health 2020;10:010369.

10 negarit.pdf. Available: https://www.moh.gov.et/ejcc/sites/default/ files/2020-04/negarit.pdf [Accessed 24 May 2021]

11 Mesele E. Preparedness and response plan for covid-19, 2020.

12 Khankeh HR, Hosseini SH, Farrokhi M, et al. Early warning system models and components in emergency and disaster: a systematic literature review protocol. Syst Rev 2019;8:315.

13 Woyessa AB, Mengesha M, Belay D, et al. Epidemiology of influenza in Ethiopia: findings from influenza sentinel surveillance and respiratory infection outbreak investigations, 2009-2015. BMC Infect Dis 2018;18:449.

14 Tadesse M, Mengesha M, Tayachew A, et al. Burden and seasonality of medically attended influenza like illness (ILI) in Ethiopia, 2012 to 2017. BMC Infect Dis 2020;20:148.

15 COVID-19. Available: https://www.ephi.gov.et/index.php/publichealth-emergency/novel-corona-virus-update [Accessed 25 Sep 2020].

16 WHO. Ethiopia publications. Available: https://www.afro.who.int/ countries/30/publications [Accessed 8 Jul 2020].

17 WHO. COVID-19 preparedness Bulletin Ethiopia|strategic partnership for IHR and health security (SPH). Available: https:// extranet.who.int/sph/news/covid-19-preparedness-bulletin-ethiopia [Accessed 13 Jul 2020]

18 Lohiniva A-L, Sane J, Sibenberg K, et al. Understanding coronavirus disease (COVID-19) risk perceptions among the public to enhance risk communication efforts: a practical approach for outbreaks, Finland, February 2020. Euro Surveill 2020;25.

19 Emergency response framework (eRF), 2nd edition. Available: https://www.who.int/publications-detail-redirect/emergencyresponse-framework-(-erf)-2nd-ed [Accessed 13 Sep 2020].

20 Getaneh Y, Getaneh Y, Yizengaw A, Adane S, et al. Global lessons and potential strategies in combating COVID-19 pandemic in Ethiopia: systematic review. Clin Oncol Res 2020:1-7. 